\title{
Troisième partie
}

\section{Mesures de gestion du risque d'apparition des maladies infectieuses aquacoles}

Les réseaux de surveillance des maladies infectieuses aquacoles constituent un excellent outil de gestion de ces maladies. Un premier article (Joly et al) présente la surveillance des maladies des mollusques par le biais de deux réseaux de surveillance, REPAMO (surveillance de l'apparition des maladies chez les coquillages des côtes françaises métropolitaines) et REPANUI (surveillance de l'apparition des maladies de l'huître perlière en Polynésie). De tels réseaux n'existent pas pour les poissons. Etant donné leur intérêt pour la gestion des élevages, l'Agence Française de Sécurité Sanitaire des Aliments (AFSSA) a décidé de mettre en place cette année un réseau de surveillance des maladies des poissons, qui bénéficiera de l'expérience des réseaux mollusques.

Parmi les mesures de gestion des maladies infectieuses piscicoles, la vaccination est celle qui séduit le plus les professionnels de terrain. Un deuxième article (Quentel et al) dresse ainsi l'état des lieux des vaccins disponibles en pisciculture. La principale limite de la vaccination tient à la difficulté d'en garantir l'efficacité lors d'administration orale malgré des tentatives d'amélioration par microencapsulation. Par ailleurs, le nombre de vaccins disponibles est très limité en raison des investissements nécessaires pour obtenir une autorisation de mise sur le marché piscicole de taille réduite. Les autovaccins peuvent aussi être utilisés, notamment en situation d'urgence, même si leurs conditions de préparation sont réglementées.

La sélection génétique d'animaux résistants est porteuse d'espoir, en particulier pour les animaux destinés à l'exportation. Un troisième article (Quillet et al) présente donc la génétique de la réponse aux pathogènes piscicoles. Néanmoins, cette sélection génétique soulève des questions, d'une part sur les éventuelles corrélations génétiques entre différentes maladies ou entre une maladie et d'autres caractères (par exemple, la résistance à la SHV montre une légère corrélation négative avec la croissance chez la truite), d'autre part sur la possibilité de mutation des agents pathogènes.

Les probiotiques, les prébiotiques et les peptides antimicrobiens pourraient à terme et en partie se substituer aux antibiotiques, notamment pour des raisons d'image des élevages et des produits mais aussi par crainte de voir se développer des antibiorésistances dans les élevages. Un dernier article (Nicolas et al) souligne donc la nécessité de soutenir des études sur ce thème dans le double objectif, d'une part d'une meilleure compréhension de leurs effets sur le système immunitaire des animaux aquatiques, d'autre part de la détermination de leur innocuité pour les animaux cibles, le consommateur et l'environnement.

Il faut également rappeler la nécessité de poursuivre des études sur l'utilisation raisonnée des antibiotiques, principal moyen thérapeutique à court et moyen terme contre certaines maladies infectieuses aquacoles, émergentes ou non.

C. Cahu, H. Pouliquen 
